\title{
Über unwirksame Verfahren gegen aufsteigende Feuchtigkeit / On ineffective measures against rising damp
}

\section{Journal Article}

Author(s):

Wittmann, F.H.

Publication date:

1995

Permanent link:

https://doi.org/10.3929/ethz-b-000423027

Rights / license:

In Copyright - Non-Commercial Use Permitted

Originally published in:

Restoration of Buildings and Monuments 1(4), https://doi.org/10.1515/rbm-1995-5045 


\title{
Über unwirksame Verfahren gegen aufsteigende Feuchtigkeit
}

\author{
F.H. Wittmann \\ Institut für Baustoffe \\ ETH Zürich, Schweiz
}

\section{Zusammenfassung}

Durch aufsteigende Feuchtigkeit werden viele Bauteile nachhaltig geschädigt. Es wird einleitend darauf hingewiesen, dass die Ursachen für die aufsteigende Feuchtigkeit bis ins einzelne bekannt sind. Wirksame Verfahren zur Bekämpfung der aufsteigenden Feuchtigkeit können danach klassifiziert werden, wie diese zugrundeliegenden Mechanismen ausser Kraft gesetzt werden. Immer wieder tauchen unwirksame Verfahren auf dem Markt auf. Die Anbieter postulieren zunächst unrealistische beziehungsweise nicht nachvollziehbare Ursachen für die aufsteigende Feuchtigkeit. Dann werden Gegenmassnahmen erdacht, die genauso phantastisch und genauso wenig wirklichkeitsnah sind. Ausgedehnte Messreihen im Labor und an unterschiedlichen Bauwerken haben die vollständige Unwirksamkeit dieser Methoden belegt.

\section{On ineffective measures against rising damp}

\begin{abstract}
Numerous buildings and structural elements are seriously damaged by rising damp. First of all, it is outlined that the physical and chemical origins of capillary rise are known in all details. Effective methods to prevent capillary rise can be grouped according to teh mechanisms of capillary rise. Nevertheless, ineffective methods appear on the market continously. In the technical documentation of these methods unrealistic origins for capillary rise are presented. As a consequence, measures against rising damp which are just as unrealistic are proposed. Some of these
\end{abstract}


illusionary concepts are briefly described. As could have been anticipated, extensive test series in the laboratory and on selected buildings have shown that these methods are totally ineffective.

\section{Einleitung}

Jährlich wird viel wertvolle Bausubstanz durch aufsteigende Feuchtigkeit zerstört. Im Bereich des Fundamentes stehen die porösen Werkstoffe des Mauerwerks wie Ziegel oder Naturstein und der Mauermörtel in Kontakt mit dem angrenzenden Erdreich. Natürliche Böden sind oft so feucht, dass erdberührte Teile des Mauerwerks nahezu wassergesättigt werden. War ursprünglich keine Horizontalsperre vorgesehen, oder ist die Sperre im Laufe der Zeit verrottet, so steigt das Wasser durch den kapillaren Sog bis zu einer Gleichgewichtshöhe im Mauerwerk an. Diese Gleichgewichtshöhe hängt einerseits von der Porengrössenverteilung im Mauerwerk und von der Verdunstungsmöglichkeit des aufsteigenden Wassers ab.

Die physikalischen Grundlagen des Saugens eines porösen Werkstoffes sind seit langem hinreichend bekannt. Dieser Vorgang kann bis ins Einzelne quantitativ beschrieben werden (siehe z.B. [1-3]). Dabei kann auch der Salzgehalt eines Wandelementes berücksichtigt werden. Mit Hilfe der numerischen Simulation ist es heute auch möglich, die Feuchtigkeits- und Salzwanderung in porösen Werkstoffen wirklichkeitsnah vorherzusagen $[4,5]$.

Die Ursachen für die aufsteigende Feuchtigkeit sind also bekannt. Damit können unterschiedliche Verfahren zur Bekämpfung der aufsteigenden Feuchtigkeit danach beurteilt werden, inwieweit sie im einzelnen in der Lage sind, den Flüssigkeitsstrom zu unterbinden oder die Ursachen für den Feuchtigkeitstransport auszuschalten. Auf dieser Basis können die heute erfolgreich eingesetzten Methoden in mindestens zwei Gruppen eingeteilt werden: mechanische Barrieren und hydrophobierte Schichten.

Typische Besispiele für mechanische Barrieren sind stufenweiser Austausch eines Mauerwerkstreifens und Ersetzen durch dichtes, praktisch nicht suagfähiges Mauerwerk oder das Durchtrennen des Mauerwerks mit Hilfe einer Mauersäge und anschliessendes Verfüllen des Schlitzes mit einer dauerhaften und dichten Masse. Ausserdem gibt es die Möglichkeit, Bleche als Trennschicht ins Mauerwerk einzurammen.

Durch entsprechend angebrachte Bohrlöcher kann eine horizontal verlaufende Schicht im Mauerwerk durch Imprägnieren hydorphobiert werden. Damit ist die kapillare Saugfähigkeit in dieser Schicht wirksam unterbrochen.

Bei der zweiten Gruppe wird lokal die Saugwirkung unterbunden, während ein Wasserdampftransport weiterhin möglich ist. Die hydrophobierte Schicht muss also hinreichend dick sein, damit der nicht unterbundene Dampftransport nicht auf die 
Dauer zu einer unerwünschten Durchfeuchtung der über der Schicht liegenden Mauerteile führt. Bei den gerade genannten Methoden hängt der Erfolg im wesentlichen von der vorausgehenden Analyse und von der fachmännischen Durchführung der Massnahme ab.

In unregelmässigen Abständen drängen aber auch Verfahren auf den Markt, deren physikalische Basis nicht nachvollziehbar ist. Diese elektrophysikalischen Verfahren erscheinen immer wieder mit leichten Variationen. Kaum je wurde ein solches Verfahren kritisch und unvoreingenommen überprüft. Es fehlen auch entsprechende Beiträge in der Fachliteratur. Wahrscheinlich weigern sich viele Versuchsanstalten, die zu einer Überprüfung in der Lage wären, sich damit auseinanderzusetzen, da sehr rasch zu erkennen ist, dass bei diesen Verfahren nicht die Ratio im Vordergrund steht. Dadurch entsteht aber eine Grauzone, die es mit sich bringt, dass auch ungeeignete Verfahren immer wieder zum Einsatz kommen. Man hat den Eindruck, dass diese Verfahren abwechselnd in unterschiedlichen Ländern Europas für eine bestimmte Periode besonders aggressiv angepriesen werden. Zur Zeit ist eine gewisse Konzentration in Österreich und Frankreich festzustellen.

In diesem kurzen Beitrag sollen zunächst vermeintliche Grundlagen einiger ungeeigneter Verfahren skizziert werden, und anschliessend werden Ergebnisse präzise durchgeführter Messreihen im Labor und an baupraktischen Objekten kurz beschrieben.

\section{Beschreibung des Verfahrens}

Liest man die technischen Unterlagen unterschiedlicher Anbieter von eletrophysikalischen Trocknungsanlagen vergleichend durch, so fällt rasch auf, dass sie sich zwar in Nuancen unterscheiden, dass aber das zugrundeliegende Prinzip, wenn man es denn so bezeichnen darf, seit mehr als zwanzig Jahren das gleiche geblieben ist. Deshalb genügt es hier, lediglich die Grundgedanken kurz zu schildern.

Zunächst wird behauptet, dass elektromagnetische Felder ursächlich mit der kapillaren Saugfähigkeit in Verbindung gebracht werden können. Steht ein Haus auf einer "Zone mit elektromagnetischer Stimulation" so ist danach mit einer besonders hohen Durchfeuchtung der Wände zu rechnen. Verstärkt wird dann die Saugwirkung, nach den Unterlagen mehrerer Anbieter, noch durch die Elektroosmose. In diesem Zusammenhang taucht der bildliche Begriff "eletroosmotischer Schub" auf.

Wenn es nun gelingt, die elektromagnetischen Wellen in einem "Kästchen" über entsprechende Spulen aufzufangen, sie dann um $180^{\circ}$ phasenverschoben wieder zu emittieren, so meint man, könnte es gelingen, das gefährliche Feld durch Interferenz quasi aufzuheben. Damit ist dann das Problem der aufsteigenden Feuchtigkeit nach diesem Denkschema für beliebig dicke Wände, auch unabhängig von deren Aufbau, 
bereits gelöst, denn wo kein Feld ist, gibt es ja keine aufsteigende Feuchtigkeit. Eine Variante geht davon aus, dass die Funkimpulse, die das Kästchen aussendet, das Wasser über Elektroosmose aus dem Mauerwerk entfernt.

Man unterscheidet noch aktive und passive Verfahren. Die aktiven benötigen einen Netzanschluss und die passiven entziehen nach Angaben der Hersteller den elektromagnetischen Feldern, die es zu anullieren gilt, die für den Betrieb notwendige Energie.

Auch einige Anbieter von elektroosmotischen Anlagen haben sich dieser Bildersprache bedient. Danach wird das aufsteigende Wasser durch ein elektrisches Feld in die Höhe getrieben. Zerstört man aber dieses Feld beispielsweise durch Kurzschluss, so fällt das Wasser wieder nach unten. Man könnte es fast so ausdrücken, eine Gruppe bemüht sich, elektromagnetische Welchselfelder zu eliminieren, während die anderen ein konstantes Feld bekämpfen. Tatsächlich wird durch die aufsteigende Feuchtigkeit ein Strömungspotential aufgebaut. Dieses bremst jedoch geringfügig den Wasserstrom. Wird es ausgeschaltet, so kann lediglich ein geringer, in den meisten Fällen nicht messbarer, Anstieg der maximalen Steighöhe erwaret werden.

Diese Vorstellungen, die immer wieder neue formuliert werden, entbehren jeder physikalischen Grundlage. Die kapillare Saugfähigkeit eines porösen Bauteils hängt gar nicht von etwa vorhandenen elektromagnetischen Feldern $a b$, und über Elektroosmose kann die kapillare Steighöhe in der Praxis nachgewiesenermassen nur minimal beeinflusst werden [6-8]. So gesehen, ist eine experimentelle Überprüfung der Wirksamkeit dieser elektrophysikalischen Methoden eigentlich überflüssig. Ein Argument, das dennoch für eine entsprechende Untersuchung spricht, und das auch immer wieder vorgebracht wird, ist jedoch, dass die Geräte ja vielleicht doch funktionieren und nur die Erklärungsversuche untauglich und zu laienhaft sind. Die im folgenden Abschnitt kurz beschriebenen Messungen wurden über mehrere Jahre hinweg durchgeführt.

\section{Experimentelle Überprüfung}

\subsection{Messungen}

Aus handelsüblichem Vollziegel $(5 \times 10 \times 27 \mathrm{~cm})$ und unter Verwendung eines historischen Kalkmörtels wurden Mauerelemente hergestellt. Nach dem Erhärten wurden diese Elemente in ein Wasserbad gestellt (siehe Abbildung). Die Feuchtigkeitsaufnahme wurde einerseits durch regelmässiges Wiegen und andererseits durch eingebaute Feuchtigkeitssonden gemessen. Das Resultat waren die typischen und zu erwartenden zeitlichen Funktionen für aufgenommene Wassermenge und Steighöhe. Nach etwa zwei Monaten waren die Mauerelemente im Gleichgewicht. Schematisch 

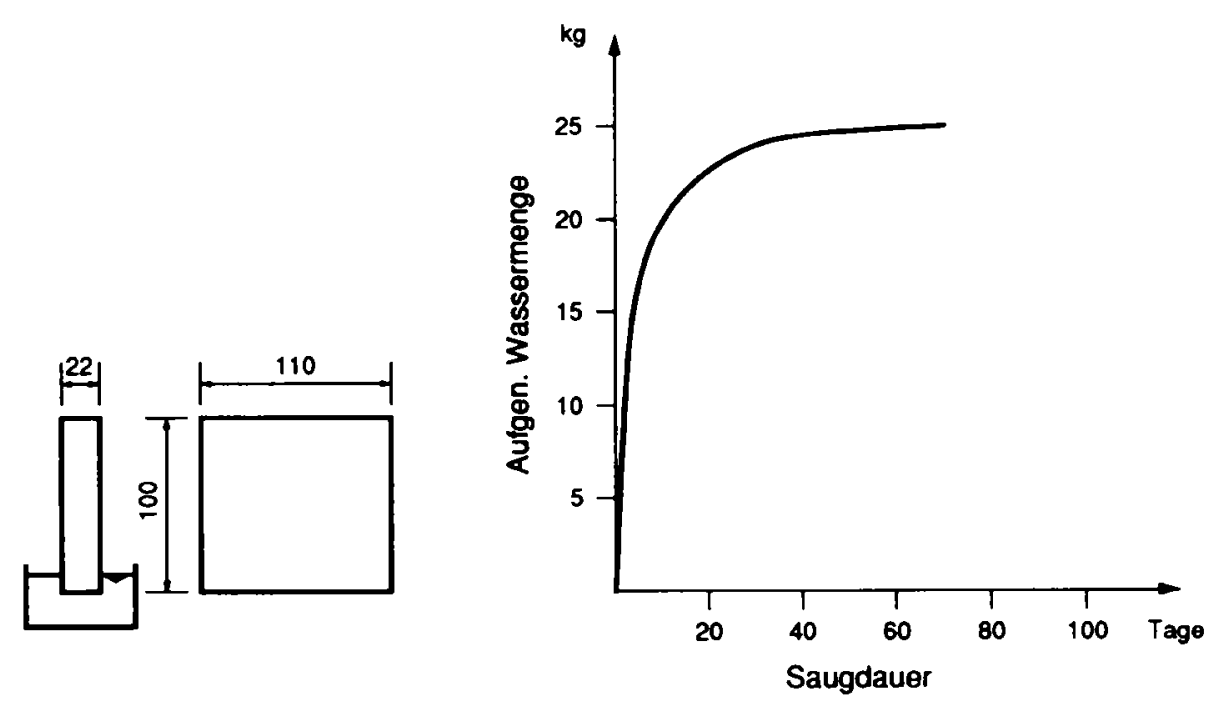

Abb: Schematische Darstellung der Mauerelemente und Wasseraufnahme als Funktion der Zeit.

Fig.: Schematic representation of the wall elements and water suction as a function ot time.

sind die verwendeten Mauerelemente und eine kapillare Saugfunktion in der Abbildung dargestellt. Dann wurde ein Gerät der nämlichen Art vom Lieferanten an einem durch ihn bestimmten Platz installiert und in Betrieb genommen. Während eines Jahres hat sich der Feuchtigkeitsgehalt in den Mauerelementen nicht messbar verändert. Sie blieben nahezu gesättigt. Geräte anderer Hersteller wurden auf die gleiche Weise und mit demselben Erfolg getestet.

\subsection{Messungen an mehreren Objekten}

Um ausschliessen zu können, dass im Labor keine aussergwöhnliche und unkontrollierbare "Zone elektromagnetischer Stimulation" herrschte, wurden an mehreren Objekten in der Praxis entsprechende Messungen durchgeführt.

Gebäude, an denen vorher bereits aufsteigende Feuchtigkeit festgestellt war, wurden ausgesucht. Im einzelnen handelte es sich um eine verputzte Aussenwand aus Ziegelstein einer Kapelle, ein hinterfülltes Natursteinmauerwerk einer Krypta und ein Natursteinmauerwerk im Kellergeschoss eines Mehrfamilienhauses. In allen Fällen wurde der Istzustand durch Entnahme von Bohrkernen und anschliessender gravimetrischer Feuchtigkeitsbestimmung festgehalten. Ein Anbieter installierte dann seine Anlagen. Der Feuchtigkeitsgehalt wurde über einen Zeitraum von mehr als 
einem Jahr durch eingebaute Sonden regelmässig verfolgt. Kleine. den jahreszeitlichen Klimaschwankungen folgende Änderungen wurden festgestellt. Zum Abschluss wurde die Feuchtigkeit noch einmal gravimetrisch an entnommenen Bohrkernen bestimmt. Das Ergebnis war ganz eindeutig, der Feuchtigkeitsgehalt blieb nach einem Jahr unverändert. So wurde experimentell nun das bestätigt, was eigentlich durch die Überlegungen schon zu erwarten war.

\section{Diskussion und Folgerungen}

Die in mehreren Varianten angebotenen elektrophysikalischen oder elektromagnetischen Verfahren zur Trocknung von Mauerwerk entbehren jeder nachprüfbaren physikalischen Grundlage. Eine Wirksamkeit ist nicht zu erwarten und konnte auch nicht experimentell nachgewiesen werden.

Für gutgläubige Bauherren und manch einen ernsthaften Denmalpfleger sind solche Geräte geradezu ein Wunschtraum. Sie erfüllen viele ihrer Forderungen, nämlich der Eingriff in die historische Bausubstanz ist minimal, die Massnahme ist reversibel und manchmal ist der Preis vergleichsweise niedrig. Nur, leider funktionieren diese Geräte nicht.

Mit einem einfachen Versuch kann die gemachte Aussage auch von Laien überprüft werden. Man stelle beispielsweise einen Ziegelstein, einen porösen Sandstein oder eine Mörtelprobe mit einer Fläche in ein Wasserbad. Die kapillare Wasseraufnahme kann an dem dunkler gefärbten, nach oben wandernden Saum mit blossem Auge beobachtet werden. Zusätzlich kann man noch die Zeit messen, die verstreicht, bis die Wasserfront am oberen Ende des Prüfkörpers ankommt und durch Wägen kann die aufgenommene Wassermenge zu diesem Zeitpunkt bestimmt werden. Danach stellt man dieselben, aber vorher wieder getrockneten, Proben wieder ins Wasserbad und umhüllt das Ganze mit einer metallischen Abschirmung gegen elektromagnetische Felder (Faraday Käfig). Sollten die elektromagnetischen Felder ursächlich mit der kapillaren Saugfähigkeit zusammenhängen, so würde unter diesen Bedingungen kein Wasser mehr aufgesaugt werden. Ein entsprechender Versuch zeigt jedoch, dass in beiden Fällen das Saugvermögen dasselbe ist. die gleiche Messung kann dann ein drittes Mal nach Installieren bzw. Einschalten eines entsprechenden Gerätes zur Bekämpfung der aufsteigenden Feuchtigkeit wiederholt werden. Sollten die Proben dann kein Wasser mehr aufnehmen, dann funktionieren diese Geräte doch. 


\section{Literatur}

/1/ W.F. Cammerer, Die kapillare Flüssigkeitsbewegung in porösen Körpern, VDIForschungsheft 500, VDI-Verlag, Düsseldorf (1963)

12/ B.H. Vos, Suction of ground water, Studies in Conservation 16, 129-144 (1971)

B/ H. Schubert, Kapillarität in porösen Feststoffsystemen, Springer Verlag, Berlin (1982)

14/ J. Prazak, J. Tywoniak und F.H. Wittmann, Experimentelle und numerische Betrachtungen zur Feuchtigkeitsbewegung in Mauerwerk, Werkstoffwissenschaften und Bausanierung, Tagungsbericht Band 1, Expert Verlag Ehningen, S. 54-56 (1993)

/5/ G. Mayer, D. Kovacova und F.H. Wittmann, Feuchtigkeitswanderung und Salztransport in Mauerwerk und Sanierputz, Sanierputsysteme, WTASchriftenreihe Heft 7, AEDIFICATIO Verlag, Freiburg i.Br., (1995)

/6/ F.H. Wittmann und W.O. Bockwijt, Grundlage und Anwendbarkeit der Eletroosmose zum Trocknen durchfeucheten Mauerwerks, Bauphysik 4, 123 127 (1982)

ก/ F.H. Wittmann, Zeta-Potential und Feuchtigkeitstransport durch poröse Werkstoffe, Werkstoffwissenschaften und Bausanierung, Edition Lack und Chemie, S. 47-50 (1983)

18/ F.H. Wittmann, S. Madra und C. Ferraris, Essais sur l'assèchement des murs à l'aide de l'électro-osmose, Chantiers Suisse, 13(7), 21-26 (1982) 
